\title{
Evaluating the use of packed cell volume as an indicator of trypanosomal infections in cattle in eastern Zambia
}

\author{
T. Marcotty ${ }^{\mathrm{a}, 1, *}$, H. Simukoko ${ }^{\mathrm{b}, 1}$, D. Berkvens ${ }^{\mathrm{a}}$, \\ J. Vercruysse ${ }^{\mathrm{c}}$, N. Praet ${ }^{\mathrm{a}}$, P. Van den Bossche ${ }^{\mathrm{a}, \mathrm{d}}$ \\ ${ }^{a}$ Institute of Tropical Medicine Antwerp, Animal Health Department, Nationalestraat 155, B-2000 Antwerp, \\ Belgium \\ ${ }^{\mathrm{b}}$ University of Zambia, School of Veterinary Medicine, Lusaka, Zambia \\ ${ }^{\mathrm{c}}$ Ghent University, Vakgroep Virologie, parasitologie en Immunologie, Salisburylaan 133, \\ B-9820 Merelbeke, Belgium \\ ${ }^{\mathrm{d}}$ Department of Veterinary Tropical Diseases, University of Pretoria, Onderstepoort, South Africa
}

Received 11 June 2007; received in revised form 23 April 2008; accepted 9 May 2008

\begin{abstract}
In this study, packed cell volume-values (PCV) are evaluated as indicator of trypanosomiasis infections in cattle. A total of 734 blood samples were collected in 11 different sampling sites in eastern Zambia: 84 calves ( $<1$ year), 52 young females and 40 young males (between 1 and 3 years), 228 cows, 317 oxen and 13 bulls ( $>3$ years). All samples were subjected to three diagnostic tests: parasitological examination using the buffy coat method, PCR/RFLP and PCV determination. The results were compared and analysed in a Bayesian model, which allowed the estimation of the infection prevalence and the respective test sensitivities and specificities. The presence of a trypanosomal infection significantly reduced the PCV, independently of the age and sex of the infected animal. The estimated prevalence of trypanosomal infections in the study area was 34\% (95\% credibility interval: 30-38\%). While the specificity of both the parasitological and the PCR/RFLP tests were set to 1 , the parasitological diagnosis had a low sensitivity (37\%) compared to the PCR/RFLP (96\%). When using a cut-off value of 24 , the PCV had a high specificity (98\%) but a rather low sensitivity (53\%) for identifying trypanosomiasis infections. Using $26 \%$ as a cut-off increased the sensitivity to $76 \%$ without much affecting the specificity (94\%). A parallel combination of the parasitological diagnosis and the PCV improved the diagnostic sensitivity ( $74 \%$ and $89 \%$ for PCV cut-off values of $24 \%$ or $26 \%$, respectively) while specificity remained high ( $98 \%$ and $94 \%$ for PCV cut-off values of $24 \%$ or $26 \%$, respectively). These results suggest that such a combination could advantageously be used for the diagnosis of cattle trypanosomiasis in the field: it is much
\end{abstract}

\footnotetext{
* Corresponding author. Tel.: +32 324762 63; fax: +32 32476263 .

E-mail address: tmarcotty@itg.be (T. Marcotty).

${ }^{1}$ Contributed equally to the work presented in this paper.
} 
more sensitive than parasitological examination alone and it is much cheaper than molecular tests. However, the value of this approach depends largely on the determination of an appropriate cut-off value to consider a sample positive, depending on the required test sensitivities and specificities.

(C) 2008 Elsevier B.V. All rights reserved.

Keywords: Trypanosomiasis; Cattle; Diagnosis; PCV; Bayesian analysis

\section{Introduction}

Trypanosomiasis is one of the main constraints to sustainable livestock production in subSaharan Africa (Swallow, 1998) where about 50-70 million animals are at risk (Geerts et al., 2001). In endemic areas, trypanosomiasis reduces calving rates, milk yields, offtake and oxen work efficiency whereas calf mortality is increased (Swallow, 2000). The disease, transmitted by tsetse flies, is caused by an infection with pathogenic trypanosomes. Such an infection causes an acute or chronic disease characterised by fever, anaemia, loss of appetite and condition. Mortality and morbidity vary widely and depend on factors such as the host species and breed, the trypanosome species, the virulence of the parasite and the innate resistance of the host (Connor and Van den Bossche, 2004).

In eastern Zambia, four trypanosome species are commonly found in cattle: Trypanosoma congolense, Trypanosoma vivax, Trypanosma brucei s.l. and Trypanosoma theileri. In the area, T. congolense and T. vivax are the most common and the most pathogenic in cattle whereas T. brucei s.l. is rare (Simukoko et al., 2007a,b) and T. theileri does not cause any disease.

Notwithstanding the availability of a number of diagnostic tests, the management of trypanosomiasis through diagnosis and treatment remains problematic (Schlater and Van den Bossche, 2004). Because of the often low parasitaemia, simple parasitological diagnostic tools such as the buffy coat method have low sensitivity and, hence, about $50 \%$ of the cases are not diagnosed and a large proportion of the infected animals remains untreated (Picozzi et al., 2002). Diagnostic problems could be resolved by using molecular diagnostic tests. These tests are highly specific and more sensitive than parasitological diagnosis (Geysen et al., 2003). Like parasitological tests, molecular tools detect the presence of the pathogens and may lack sensitivity in chronic cases when parasitaemia is low. Molecular tests require sophisticated infrastructure, are expensive and results become available after a considerable delay. They are thus less appropriate for use in rural areas where communities are mostly interested in identifying infected animals that require treatment at the cheapest price. Serological anti-trypanosomal antibody detection tests, on the other hand, are much cheaper but require laboratory facilities and positive cases are poorly associated with infection because of the persistence of the antibodies even after an infection has been cured (Van den Bossche et al., 2000). In addition, serological tests have no use in the early stages of the infection, before the rise of specific antibodies. There is thus a need for simple tools that improve the sensitivity of the parasitological diagnosis, particularly in chronic clinical cases, and hence support the management of trypanosomiasis.

In cattle and other susceptible domestic animals, anaemia is a well-recognized and inevitable consequence of an infection with pathogenic trypanosomes, including T. congolense and T. vivax (Murray and Dexter, 1988). It is measured by determining the packed cell volume (PCV). In the absence of other factors causing anaemia, the PCV gives a reliable indication of the disease status of a trypanosome-infected animal (Trail et al., 1993; Grace et al., 2007). Hence, in the absence of other 
factors causing anaemia, the use of PCV and its parallel combination with the parasitological diagnosis could improve the detection of trypanosome-infected animals and ease the decision to treat them with a trypanocidal drug. Parallel combinations of diagnostic tests, for which one single positive test makes a combination positive, should however be used with caution. While their sensitivity is higher than those of the individual diagnostic tests, their specificity is lower.

The objective of this study was to evaluate the use of PCV and determine its sensitivity and its specificity, in the diagnosis of bovine trypanosomiasis, either on its own or in combination with parasitological examination. In the absence of gold standards (100\% sensitive and specific tests), Bayesian models are useful to evaluate the disease prevalence and the characteristics of batteries of tests applied on the same sets of samples (Lesaffre et al., 2007). These iterative models integrate data and prior information to make estimates. The prior information consists of the conditional probabilities underlying the relationship between the various test results and the disease prevalence and the limits within which these parameters are allowed to vary. Prior information should be sufficiently detailed to allow the convergence of the models but it should be kept in mind that priors have an important effect on their output. Priors are usually based on expert's opinion. It is however acknowledged that such opinion may lack objectivity.

\section{Materials and methods}

The study was conducted in a trypanosomiasis endemic area of eastern Zambia. The area was chosen because of the high prevalence of trypanosomal infections in cattle and the absence of tick-borne diseases that could cause anaemia in animals.

\subsection{Study area}

The Eastern Province of Zambia lies between latitudes $10^{\circ}$ to $15^{\circ}$ South and longitude $30^{\circ}$ to $33^{\circ}$ East. The study took place in the Katete and Petauke districts, north of the Great East Road, at an altitude ranging between 900 and $1000 \mathrm{~m}$ above sea level. East Coast fever does not occur in the region and, although other tick-borne pathogens such as Babesia spp. and Anaplasma spp. are present, the diseases are endemically stable, causing virtually no clinical cases in cattle. Trypanosomiasis transmitted by Glossina morsitans morsitans is also endemic. The prevalence of trypanosome infections differs substantially between livestock species with cattle being the most important reservoir of infection (Simukoko et al., 2007a).

\subsection{Sample selection}

A cross-sectional survey was conducted at 11 randomly selected sampling sites (crushpens) during the dry season (Simukoko et al., 2007a). The sampling sites were selected in a lottery from a sampling frame of all the crush pens with more than 100 heads of cattle. The list of crush pens and the cattle population data were provided by the provincial veterinary office. All animals belonged to the Angoni breed and were maintained in a traditional husbandry system. The animals mainly feed by grazing, assuring appropriate nutritional status ( 4 or above on the 9 level scale of Nicholson and Butterworth, 1986), including in the dry season during which they are left in free range. The sample size in the sampling sites was calculated to provide $95 \%$ certainty of detecting at least one positive case at a prevalence of 5\% (Thrusfield, 1995). A prevalence of 5\% or less would be considered as marginal. The calculated sample size was 59. In each sampling

site, animals were recorded and allocated to the different age and sex classes. Systematic 
sampling was then performed in such a way that the number of samples in each age and sex category was proportional to the herd structure recorded in the sampling site. This was done to assure a good representativeness of the samples, since age and sex are known to affect the exposure to trypanosomoiasis (Simukoko et al., 2007b). A total of 734 blood samples were collected (between 57 and 83 in each crushpen): 84 calves ( $<1$ year old), 52 young females and 40 young males (between 1 and 3 years of age), 228 cows, 317 oxen and 13 bulls ( $>3$ years).

\subsection{Blood collection and diagnosis}

From each selected animal, jugular blood was collected in tubes with EDTA as anticoagulant. After sampling, the tubes were placed in a box containing ice packs and transported to the laboratory within $4 \mathrm{~h}$ of collection.

From each tube, blood was transferred into three capillary tubes. The capillary tubes were spun in a microhaematocrit centrifuge for $5 \mathrm{~min}$ at $9000 \mathrm{rpm}$. After centrifugation, the packed cell volume (PCV) was determined. The buffy coat and the uppermost layer of red blood cells of each specimen were extruded onto a microscope slide and examined for the presence of motile trypanosomes. Samples were examined with a phase-contrast microscope at $400 \times$ magnification (Murray et al., 1977). At least 50 fields were observed before declaring a slide as negative. Blood samples that were positive were further processed as blood smears for trypanosome species identification. Giemsa-stained thick and thin blood smears were examined under $100 \times$ oil immersion objective lens $(1000 \times$ magnification) to obtain a parasitological diagnosis.

The buffy coats of the two remaining capillary tubes were extruded onto a labelled filter paper (Whatman no. 3, Whatman ${ }^{\circledR}$ ). Filter papers were stored in sealed plastic bags containing silica gel and transferred in a freezer at $-18{ }^{\circ} \mathrm{C}$. The samples were further analysed using the PCR/RFLP described by Geysen et al. (2003) to detect Trypanosoma spp. DNA. This test, which detects 1 genome in $40 \mu \mathrm{l}$ of blood, allows the identification of T. congolense, T. brucei, T. vivax and T. theileri with a high degree of specificity (Geysen et al., 2003). Briefly, the protocol was as follows. Phenol extraction was used to extract the DNA from the filter papers. Standard PCR amplifications were carried out in $25-\mu \mathrm{l}$ reaction mixtures containing $5-\mu 1$ sample, $50 \mathrm{mM} \mathrm{KCl,} 10 \mathrm{mM}$ Tris-HCl (pH 8.3), $1.5 \mathrm{mM} \mathrm{MgCl}_{2}, 200-\mu$ l of each dNTP, 20 pmol of each primer and $0.5 \mathrm{U}$ Taq polymerase enzyme (Goldstar, Eurogentec). After a denaturation step of 4 min at $94{ }^{\circ} \mathrm{C}$ each of the 40 cycles consisted of $45 \mathrm{~s}$ at $92{ }^{\circ} \mathrm{C}, 45 \mathrm{~s}$ at $58{ }^{\circ} \mathrm{C}$ and $60 \mathrm{~s}$ at $72{ }^{\circ} \mathrm{C}$. Semi-nested runs were performed in which $0.5 \mu \mathrm{l}$ of amplification product from the first run was added to $24.5 \mu \mathrm{l}$ of PCR mix, containing the same ingredients and concentrations except for 25 cycles. The first amplification was done on the $18 \mathrm{~S}$ gene using the forward primer 18ST nF2 (CAACGATGACACCCATGAATTGGGGA) and 18ST nR3 (TGCGCGACCAATAATTGCAATAC) as reverse primer. A semi-nested second amplification was done using the forward primer 18ST nF2 of the first amplification with the reverse primer 18ST nR2 (GTGTCTTGTTCTCACTGACATTGTAGTG).

For further typing of the fragments, RFLP-based methods were used. Nested products were digested with Msp1 and Eco571 enzymes in buffer Y+/Tango with $S$-adenosylmethionine according to the manufacturer's specifications (Gibco, UK) using 6- $\mu$ l of amplified DNA in $15-\mu$ l total volume.

\subsection{Data analysis}

Parasitological and PCR/RFLP results were considered positive when either T. congolense or T. vivax were present in a single or mixed infection. 
The PCV-values were first analysed using a robust linear regression in Stata 9 (StataCorp, 2006) to detect a possible confounding effect of the age and sex category or an effect of trypanosomal infections on the PCV. The indicator used for trypanosomal infection was the PCR/ RFLP result since it is currently the most sensitive and specific tool available for the diagnosis of trypanosomiasis in cattle (Geysen et al., 2003). Explanatory variables were the PCR/RFLP binary results, the age and sex category, and the interaction between the two (PCV $=$ a.PCR + b.category + c.PCR.category). Primary sampling units (sampling sites) and strata (age and sex classes) were taken into account. The significance of grouped explanatory variables was evaluated using the adjusted Wald test. The distribution of the residuals was verified by plotting their quantiles against the quantiles of the normal distribution (Q-Q plot). Heteroskedasticity was tested $(P>0.05)$ in non-robust models using the same explanatory variables.

In view of quickly estimating the PCV-values which could be of any use in the diagnosis of trypanosomiasis, PCV-values were transformed in binary data using cut-off values ranging from the smallest to the largest observation. The sensitivity and the specificity of each transformation were calculated, using the PCR/RFLP results as a reference and plotted in a receiver operator characteristic (ROC) curve.

Finally, the binary results of the PCR/RFLP test, the parasitological diagnosis and the PCV (using defined cut-off values) were introduced in a Bayesian model to estimate the sensitivity and the specificity of the three diagnostic tests (Berkvens et al., 2006). The Bayesian model was run in Winbugs (http://www.mrc-bsu.cam.ac.uk/bugs). The model (Appendix A) contained conditional probabilities, which were restricted as follows:

- The specificity of the parasitological diagnosis and the PCR/RFLP were set to 1 ; it is acknowledged that the true specificities of these tests can only be less than 1 but the specificity of both the parasitological (Schlater and Van den Bossche, 2004) and the PCR/REFLP (Geysen et al., 2003) tests were reported to be close to 1 .

- The sensitivity of the PCR/RFLP was allowed to range between 0.9 and 1 ; this is in accordance with previously reported observations (Geysen et al., 2003) and it reflects the high sensitivity of the technique.

- Based on our own experience, the conditional probability that an infected animal testing negative at PCR/RFLP would test positive at the parasitological diagnosis was set to a maximum of 0.1 ; this is based on the fact that both test sensitivities depend on the level of parasitaemia and on the postulation that an infected animal that is negative at PCR/RFLP is unlikely to be positive at a parasitological examination.

The conformity of the model was evaluated using the criteria proposed by Berkvens et al. (2006). In brief, the deviance information criterion (DIC) and the effective number of parameters estimated (pD) were calculated using, on one hand, parent node values in Winbugs and, on the other hand, the posterior means of the multinomial probabilities in $R$ (http://www.r-project.org) (Appendix B). The agreement between the two types of calculations provides assurance on the conformity of the model. A Bayes $P$ value (Gelman et al., 2003) close to 0.5 is another indicator of conformity. A high Bayes $P$ value denotes a lack of fit of the model with the data. Each model was run three times in Winbugs. First, a non-informative model, without any constraint on the conditional probabilities was run to estimate the DIC and pD. Second, the genuine model with the constraints listed above was run and the Bayes $P, \mathrm{DIC}$ and $\mathrm{pD}$ values were evaluated. This model provided estimates of the conditional probabilities and credibility intervals. Finally, a validation model, with strict constraints applied around the conditional probability estimates, was run to 
confirm that the Bayes $P$ values tended towards 0 . For each model, 20,000 iterations were used in three chains, following a burn-in of 10,000. Convergence was ensured by examining plots of the variable values against iteration numbers for the three chains. The sensitivity and the specificity of the three diagnostic tests as well as those of the combination of the parasitological test and the PCV were estimated.

\section{Results}

Clear discrepancies were observed between the results of the parasitological and PCR/RFLP diagnoses. Twenty percent of the samples (149/734) were positive on PCR/RFLP and negative at parasitological examination while none of the parasitologically positive samples were negative on PCR/RFLP. Since the specificity of both tests is assumed to be one, this reflects a significant difference in the sensitivity of the two tests (Fisher exact test: $p<0.001$ ).

Taking the PCR/RFLP results as a reference, the distribution of the PCV-values of positive and negative animals differed substantially (Fig. 1). The linear regression of the PCV-values indicated a significant effect of the PCR/RFLP results $(p<0.001)$ but no effect of the age and sex category of cattle from which the samples originated and their interaction with PCR/RFLP (grouped adjusted Wald test: $p=0.87$ ). The ROC curve (Fig. 2) shows that, in the study area, the PCV-value that is usually used as a cut-off for anaemia in cattle $(<24 \%)$ has a high specificity but a rather low sensitivity for identifying Trypanosoma spp PCR/RFLP positive animals $(0.97$ and 0.54 , respectively). Using a cut-off of $26 \%$ increases the sensitivity to 0.77 without much affecting the specificity (0.93).

The data used in the two Bayesian models, with $24 \%$ and $26 \%$ as cut-off values for the transformation of the PCV-values in binomial values, are presented in Table 1 . The indicators of the Bayesian analyses are presented in Appendices C and D. They show a good fit of the data with the experts' constraints (Berkvens et al., 2006).

The conditional probabilities estimated by the two Bayesian models are listed in Appendix E. Among them, theta[1] (prevalence), theta[4] (parasitology sensitivity related parameter), theta[8] and theta[9] (PCV sensitivity related parameters) and theta[12] (PCV specificity related parameter) were well estimated, with narrow confidence intervals. For the others, the posterior distribution ranged uniformly between the limits of the constrained intervals, either because of

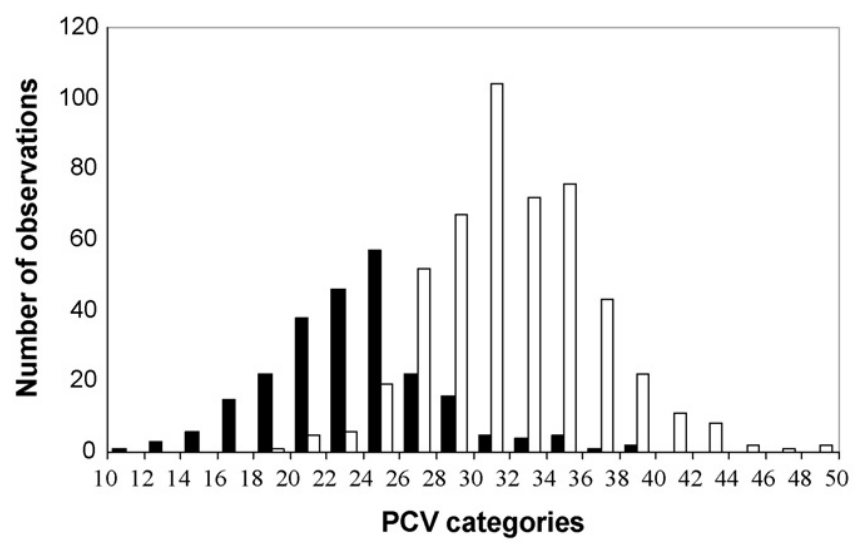

Fig. 1. PCV-distribution of PCR/RFLP positive ) and negative $(\square)$ animals. 


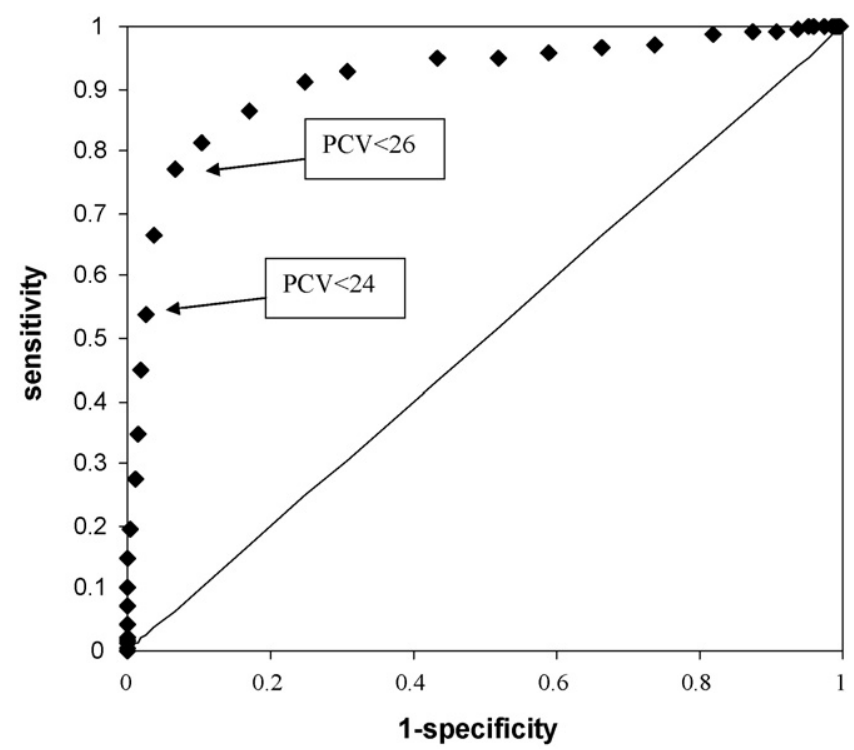

Fig. 2. ROC curve of PCV binary results using various cut-off values ( $<10$ to $<48$, step 1$)$; the PCR/RFLP test was considered as the reference test.

the severity of the constraints (e.g.: theta[2], theta[3], theta[5], theta[6] and theta[7]) or because of a lack of data (e.g.: given its high sensitivity, very few infected animals are negative at the PCR/RFLP test, implying that theta[10] and theta[11] are poorly estimated).

According to these models, the prevalence of trypanosomal infections was $34 \%(95 \%$ credibility interval: $31-39 \%$ ). The models confirmed the low sensitivity of the parasitological diagnosis compared to the PCR/RFLP test (37\% and 96\%, respectively). They also indicated that the PCV has a better sensitivity than the parasitological diagnosis, whereas its estimated specificity was over $94 \%$ (Fig. 3). A parallel combination of the parasitological diagnosis and the PCV improves diagnostic sensitivity while specificity remains high (Fig. 3). The estimated sensitivity of test combinations is higher than for independent test combinations $\left[1-\left(1-\mathrm{se}_{\mathrm{para}}\right)\left(1-\mathrm{se}_{\mathrm{pcv}}\right)=0.70\right.$ and 0.85 for $<24$ and $<26$ cut-offs, respectively $]$. The

Table 1

Number of observations recorded in the eight categories corresponding to a combination of the three test results and depending on the PCV cut-off value

\begin{tabular}{llllr}
\hline PCR/RFLP & Parasitology & PCV & \multicolumn{2}{c}{ Number of observations } \\
\cline { 4 - 5 } & & & PCV $<24$ & PCV $<26$ \\
\hline- & - & - & 479 & 460 \\
- & - & + & 12 & 31 \\
- & + & - & 0 & 0 \\
- & + & + & 0 & 0 \\
+ & - & + & 61 & 22 \\
+ & - & - & 88 & 127 \\
+ & + & + & 51 & 33 \\
+ & + & & 43 & 61 \\
Total & & & 734 & 734 \\
\hline
\end{tabular}


(A). PCV cut-off: $<24$

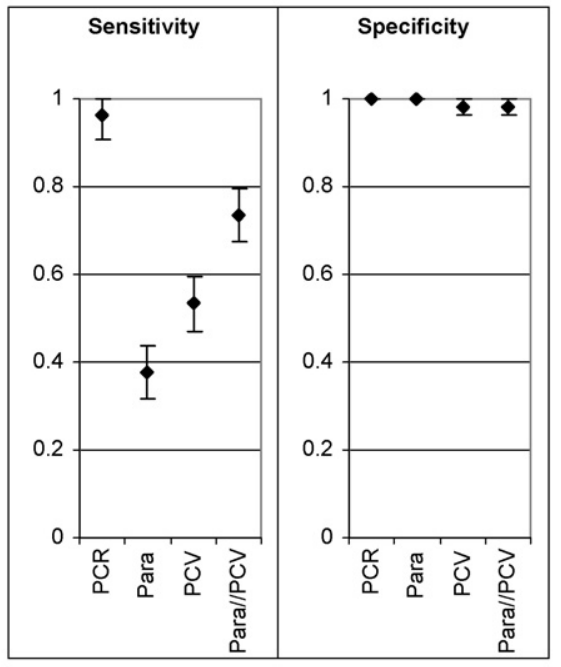

\begin{tabular}{lll}
\hline PCR & $0.96(0.91-1)$ & $1(1-1)$ \\
Para & $0.37(0.31-0.44)$ & $1(1-1)$ \\
PCV & $0.53(0.47-0.6)$ & $0.98(0.96-1)$ \\
Para//PCV & $0.74(0.67-0.79)$ & $0.98(0.96-1)$ \\
\hline
\end{tabular}

(B). PCV cut-off: $<26$

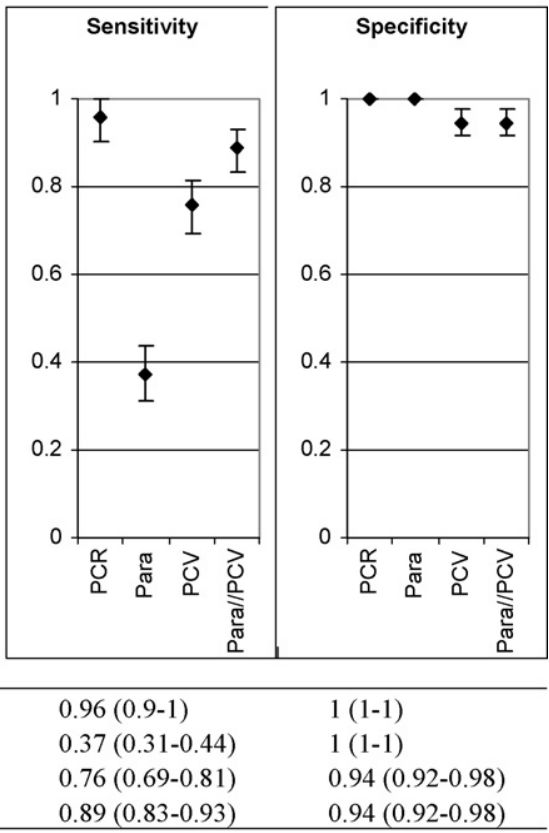

Fig. 3. Estimated sensitivity and specificity and their $95 \%$ credibility intervals for the parasitological test (Para), the PCR/ RFLP test (PCR), the PCV and the parallel combination of the parasitological test and the PCV (Para/PCV) using two different PCV cut-off values (24 in A and 26 in B).

specificity is lower than that of the parasitological and the PCR/RFLP tests, which are assumed to be $100 \%$.

\section{Discussion}

The results of the study confirm the low sensitivity of the routinely used parasitological tests for the diagnosis of trypanosomiasis. Despite the concentration of the parasite, the buffy coat method fails to detect $66 \%$ of the infected animals in the study area. This finding confirms many earlier observations and is attributed to the low parasitaemia especially in chronic trypanosomiasis cases (Picozzi et al., 2002). However, in the absence of other diseases causing anaemia in individual animals, the findings of this study suggest that the PCV-value of an individual animal is a good indicator of the presence of a trypanosomal infection. This is especially the case when the PCV-value is used jointly with the results of the parasitological diagnosis. Indeed, by combining the two types of information, the diagnostic sensitivity increases to $74 \%$ and even $89 \%$ using a PCV cut-off of $24 \%$ or $26 \%$, respectively, which is considerably higher than the sensitivity of the parasitological diagnosis on its own. These values are surprisingly higher than in independent test combinations. This is related to the fact that the conditional probability theta 8 is lower than theta 9 in Appendix E. This implies a negative dependence between the two diagnostic tests in infected animals: a parasitologically positive animal is less likely to have anaemia than an infected parasitologically negative animal. This could be explained by the fact that early infections are more likely to be detected using 
parasitological diagnostic tools even before the development of anaemia whereas chronically infected animals, usually presenting low parasitaemia, are more likely to be anaemic.

Although both the sensitivity and the specificity of the parallel combination of the PCV and the parasitological test is lower than that of the PCR/RFLP, the cost of the former is a fraction of the costs involved when conducting PCR/RFLP. Moreover, the equipment required for parasitological diagnosis and PCV measurement should be part of the standard equipment of field veterinary laboratories in trypanosomiasis endemic areas. Since under the conditions prevailing in the study area, the PCV-value on its own already has a high diagnostic sensitivity and specificity, one could consider the use of a simple tool to be applied easily by animal health technicians or cattle owners to assess the level of anaemia of an animal. FAMACHA ${ }^{(}$ eye color chart and hemoglobin color scale tests, which showed evidence of robustness and reliability for the detection of anaemia in cattle in western Africa (Grace et al., 2007), could possibly be used as an alternative to PCV measurement in spite of a probable lower precision. In fact, similar tools have been implemented successfully in the control of hemonchosis in sheep and goats (Bath et al., 2001).

The applicability of this simple approach relies heavily on the level of anaemia at which an animal is considered to be infected with trypanosomes. The analysis of a ROC curve, using PCR/ RFLP results as a reference should help in selecting the most appropriate cut-off, depending on the required test sensitivity and specificity. The specificity of the parallel combination of PCV and parasitological diagnosis could be affected by infections with Anaplasma spp., Babesia spp. or Theileria parva (Fandamu et al., 2007), by gastro-intestinal worm infestations (Grace et al., 2007) or by any other factor which could cause anaemia in cattle. Good nutritional status was reported to reduce the impact of trypanosome infection on PCV (Holmes et al., 2000; Van den Bossche and Rowlands, 2001). This could reduce the sensitivity of the PCV as indicator of trypanosomal infections in animals with high nutritional status. However, animals' nutritional state is unlikely to affect its specificity since anaemia of nutritional origin can only be caused by severe starvation and nutritional deficiencies (Anon., 2006).

In situations where other causes of anaemia are suspected, additional indicators, specific for these causes (parasitaemia, coprological detection of eggs from helminths that may cause anaemia i.e. Haemonchus, Fasciola..., fever and other specific clinical signs), might be considered to discriminate their effect on the PCV from that of trypanosomiasis. In any case, the specificity of the proposed diagnostic approach should be confirmed and monitored in areas where it is to be used, for instance by comparing a sample of the results to a PCR/RFLP analysis in a ROC curve.

In conclusion, the clinical evaluation of anaemia may be a useful low-cost adjunct to other trypanosomiasis management tools. It would certainly complement the parasitological tests and provide the animal health workers that do not have diagnostic tools at their disposal with a relatively objective tool that can be used in identifying animals that require treatment with trypanocidal drugs.

\section{Acknowledgements}

This study was supported by the Flemish Inter University Council (Belgium). The authors would like to thank the Department of Veterinary Services of Katete District of the Eastern Province of Zambia for use of the laboratory, the livestock owners for providing the animals for the study, Mrs Siberia Banda and Mr. Mwango for field and laboratory assistance and the School of Veterinary Medicine of the University of Zambia for providing laboratory space. 


\section{Appendix A. Bayesian model run in Winbugs}

\{

$\mathrm{N}<-\operatorname{sum}(\mathrm{r}[])$

$r[1: 8] \sim d m u l t i(p[1: 8], N)$

$\mathrm{p}[1]<-$ theta[1]* $(1-\text { theta[2] })^{*}(1-\text { theta[5] })^{*}(1-$ theta[11] $)+\left(1-\right.$ theta[1])*theta[3]*theta[6 ${ }^{*}$ theta[12]

$\mathrm{p}[2]<-$ theta[1] ${ }^{*}(1-\text { theta[2] })^{*}(1-\text { theta[5] })^{*}($ theta[11] $)+(1-\text { theta[1] })^{*}$ theta[3]*theta[6]* $(1-$ theta[12])

$\mathrm{p}[3]<-$ theta[1]* $(1-\text { theta[2] })^{*}$ theta[5]* ${ }^{*}(1-$ theta[10] $)+\left(1\right.$-theta[1])*theta[3]* ${ }^{*}$ 1-theta[6])*theta[13]

$\mathrm{p}[4]<-$ theta[1] ${ }^{*}(1-\text { theta[2] })^{*}$ theta[5]* $($ theta[10] $)+(1-\operatorname{theta}[1])^{*}$ theta[3] ${ }^{*}(1-\text { theta[6] })^{*}(1-$ theta[13] $)$

$\mathrm{p}[5]<-$ theta[1]*theta[2] ${ }^{*}(1-\text { theta[4] })^{*}(1-$ theta[9] $)+(1-\operatorname{theta}[1])^{*}(1-\text { theta[3] })^{*}$ theta[7] ${ }^{*}$ theta[14]

$\mathrm{p}[6]<-$ theta[1]*theta[2]* $(1-\text { theta[4] })^{*}(\text { theta[9])+(1-theta[1] })^{*}(1-\text { theta[3] })^{*}$ theta[7]* $(1-$ theta[14])

$\mathrm{p}[7]<-$ theta[1] ${ }^{*}$ theta[2] $]^{*}$ theta[4]* $(1-$ theta[8] $)+(1-\text { theta[1] })^{*}(1-\text { theta[3] })^{*}(1-\text { theta[7] })^{*}$ theta[15]

$\mathrm{p}[8]<-$ theta[1]*theta[2]*theta[4]* $]^{*}$ theta[8])+(1-theta[1] $)^{*}(1-\text { theta[3] })^{*}(1-\text { theta[7] })^{*}(1-$ theta[15])

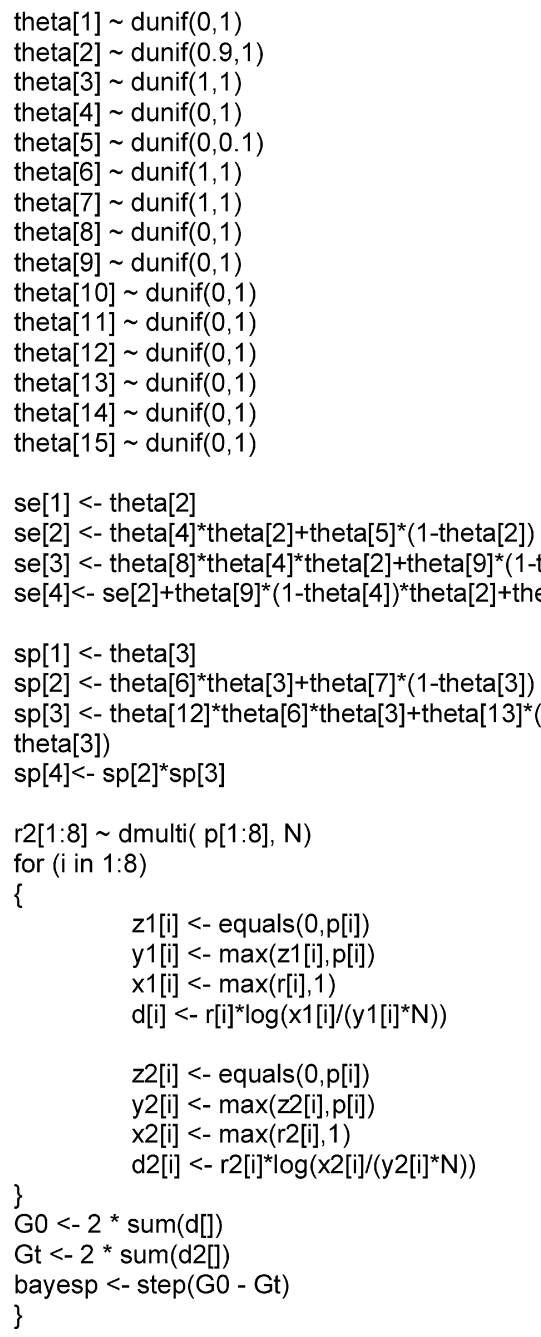

$\mathrm{p}[1: 8]$ are the number of observations of each of the test result combinations (Table 1); theta[1:15] are the conditional probabilities (see Appendix E) subjected to experts' constraints; se[1:4] are the sensitivity of the PCR/RFLP, the parasitological test, the PCV and the parallel combination of the parasitological test and the PCV; sp[1:4] are the specificity of the same tests. 


\section{Appendix B. Code for the estimation of DIC and pD using posterior means of the multinomial probabilities in $R$ software}






\section{Appendix C. DIC and pD values estimated using parent nodes and posterior means and obtained from the bayesian models ran on the two datasets with different constraints applied on the parameters}

\begin{tabular}{|c|c|c|c|c|}
\hline & \multicolumn{2}{|l|}{$\mathrm{PCV}<24$} & \multicolumn{2}{|l|}{$\mathrm{PCV}<26$} \\
\hline & Using parent nodes & $\begin{array}{l}\text { Using posterior means of } \\
\text { the multinomial probabilities }\end{array}$ & $\begin{array}{l}\text { Using parent } \\
\text { nodes }\end{array}$ & $\begin{array}{l}\text { Using posterior means of } \\
\text { the multinomial probabilities }\end{array}$ \\
\hline \multicolumn{5}{|c|}{ Model without constraints } \\
\hline DIC & -365.80 & 40.82 & -299.25 & 41.00 \\
\hline $\mathrm{pD}$ & -401.74 & 4.88 & -335.38 & 4.87 \\
\hline \multicolumn{5}{|c|}{ Model with experts' constraints } \\
\hline DIC & 38.00 & 38.12 & 38.26 & 38.33 \\
\hline $\mathrm{pD}$ & 4.73 & 4.85 & 4.80 & 4.87 \\
\hline
\end{tabular}

DIC and $\mathrm{pD}$ values of the constrained models are similar whether their estimation is based on parent nodes or on posterior means of the multinomial probabilities, unlike the unconstrained model; in both models restricted by experts' opinion, pD values, which correspond to the number of parameters estimated in Bayesp models, are close to the maximum (estimated in the unconstrained models using posterior means).

\section{Appendix D. Bayesp values obtained from the Bayesian models ran on the two datasets with different constraints applied on the parameters}

\begin{tabular}{lll}
\hline & PCV $<24$ & PCV $<26$ \\
\hline Model without constraints & 0.61 & 0.61 \\
Model with experts' constraints & 0.48 & 0.48 \\
Model constrained on estimates & 0.004 & 0.0007 \\
\hline
\end{tabular}

Bayesp values indicate too severe constraints when diverting from 0.5; Bayesian value tend towards 0 when appropriate constraints are applied.

\section{Appendix E. Conditional probabilities (and their $95 \%$ credibility intervals) estimated by the Bayesian models}

\begin{tabular}{lllll}
\hline Code & Conditional probabilities & Constraints & PCV $<24$ & PCV $<26$ \\
\hline theta[1] & $\operatorname{Pr}\left(D^{+}\right)$ & & $0.34(0.31-0.38)$ & $0.35(0.31-0.39)$ \\
theta[2] & $\operatorname{Pr}\left(T_{1}^{+} \mid D^{+}\right)$ & $0.9-1$ & $0.96(0.91-1)$ & $0.96(0.9-1)$ \\
theta[3] & $\operatorname{Pr}\left(T_{1}^{-} \mid D^{-}\right)$ & $=1$ & $1(1-1)$ & $1(1-1)$ \\
theta[4] & $\operatorname{Pr}\left(T_{2}^{+} \mid D^{+} \cap T_{1}^{+}\right)$ & & $0.39(0.33-0.45)$ & $0.39(0.33-0.45)$ \\
theta[5] & $\operatorname{Pr}\left(T_{2}^{+} \mid D^{+} \cap T_{1}^{-}\right)$ & $0-0.1$ & $0.04(0-0.1)$ & $0.04(0-0.1)$ \\
theta[6] & $\operatorname{Pr}\left(T_{2}^{-} \mid D^{-} \cap T_{1}^{-}\right)$ & $=1$ & $1(1-1)$ & $1(1-1)$ \\
theta[7] & $\operatorname{Pr}\left(T_{2}^{-} \mid D^{-} \cap T_{1}^{+}\right)$ & $=1$ & $1(1-1)$ & $1(1-1)$ \\
theta[8] & $\operatorname{Pr}\left(T_{3}^{+} \mid D^{+} \cap T_{1}^{+} \cap T_{2}^{+}\right)$ & & $0.46(0.36-0.56)$ & $0.65(0.55-0.74)$ \\
theta[9] & $\operatorname{Pr}\left(T_{3}^{+} \mid D^{+} \cap T_{1}^{+} \cap T_{2}^{-}\right)$ & & $0.59(0.51-0.67)$ & $0.85(0.79-0.9)$ \\
theta[10] & $\operatorname{Pr}\left(T_{3}^{+} \mid D^{+} \cap T_{1}^{-} \cap T_{2}^{+}\right)$ & & $0.5(0.03-0.98)$ & $0.5(0.03-0.98)$ \\
theta[11] & $\operatorname{Pr}\left(T_{3}^{+} \mid D^{+} \cap T_{1}^{-} \cap T_{2}^{-}\right)$ & & $0.46(0.02-0.97)$ & $0.5(0.02-0.97)$ \\
theta[12] & $\operatorname{Pr}\left(T_{3}^{-} \mid D^{-} \cap T_{1}^{-} \cap T_{2}^{-}\right)$ & & $0.98(0.96-1)$ & $0.94(0.92-0.98)$ \\
theta[13] & $\operatorname{Pr}\left(T_{3}^{-} \mid D^{-} \cap T_{1}^{-} \cap T_{2}^{+}\right)$ & & $0.5(0.02-0.98)$ & $0.5(0.03-0.98)$ \\
theta[14] & $\operatorname{Pr}\left(T_{3}^{-} \mid D^{-} \cap T_{1}^{+} \cap T_{2}^{-}\right)$ & & $0.5(0.02-0.97)$ & $0.5(0.02-0.97)$ \\
theta[15] & $\operatorname{Pr}\left(T_{3}^{-} \mid D^{-} \cap T_{1}^{+} \cap T_{2}^{+}\right)$ & & $0.5(0.03-0.97)$ & $0.5(0.02-0.98)$ \\
\hline$T_{1}, T_{2}$ and & & &
\end{tabular}

$T_{1}, T_{2}$ and $T_{3}$ are the PCR/RFLP, the parasitological test and the PCV, respectively 


\section{References}

Anon., 2006. The Merck Veterinary Manual. Merck \& Co. Inc., Whitehouse Station.

Bath, Hansen, Krecek, Van Wyk, Vatta, (Eds.), 2001. Sustainable approaches for managing haemonchosis in sheep and goats. FAO Animal Production and Health Paper. FAO, Rome.

Berkvens, D., Speybroeck, N., Praet, N., Adel, A., Lesaffre, E., 2006. Estimating disease prevalence in a Bayesian framework using probabilistic constraints. Epidemiology 17, 145-153.

Connor, R.J., Van den Bossche, P., 2004. African animal trypanosomoses. In: Coetzer, J.A.W., Tustin, R.C. (Eds.), Infectious Diseases of Livestock. Oxford University Press southern Africa, Cape Town, pp. 251-296.

Fandamu, P., Marcotty, T., Brandt, J.R.A., Duchateau, L., Speybroeck, N., Dolan, T.T., Berkvens, D., 2007. Red blood cell volume as a predictor of fatal eactions in cattle infected with Theileria parva Katete. Onderstepoort. J. Vet. Res. 74, 37-43.

Geerts, S., Holmes, P.H., Eisler, M.C., Diall, O., 2001. African bovine trypanosomiasis: the problem of drug resistance. Trends Parasitol 17, 25-28.

Gelman, Carlin, Stern, Rubin, (Eds.), 2003. Bayesian Data Analysis. Chapman \& Hall/CRC, Boca Raton.

Geysen, D., Delespaux, V., Geerts, S., 2003. PCR-RFLP using Ssu-rDNA amplification as an easy method for speciesspecific diagnosis of Trypanosoma species in cattle. Vet. Parasitol. 110, 171-180.

Grace, D., Himstedt, H., Sidibe, I., Randolph, T., Clausen, P.H., 2007. Comparing FAMACHA eye color chart and hemoglobin color scale tests for detecting anemia and improving treatment of bovine trypanosomosis in West Africa. Vet. Parasitol. 147, 26-39.

Holmes, P.H., Katunguka-Rwakishaya, E., Bennison, J.J., Wassink, G.J., Parkins, J.J., 2000. Impact of nutrition on the pathophysiology of bovine trypanosomiasis. Parasitology 120 (Suppl), S73-S85.

Lesaffre, E., Speybroeck, N., Berkvens, D., 2007. Bayes and diagnostic testing. Vet. Parasitol. 148, 58-61.

Murray, M., Dexter, T.M., 1988. Anaemia in bovine African trypanosomiasis: a review. Acta Trop. 45, 389-432.

Murray, M., Murray, P.K., McIntire, W.M., 1977. An improved parasitological techniques for the diagnosis of African trypanosomiasis. Trans. Roy. Soc. Trop. Med. Hyg. 71, 325-326.

Nicholson, Butterworth (Eds.), 1986. A Guide to Body Condition Scoring of Zebu Cattle. International Livestock Center for Africa (ILCA), Addis Ababa, Ethiopia.

Picozzi, K., Tilly, A., Fèvre, E.M., Coleman, P., Magona, J.W., Odiit, M., Eisler, M.C., Welbum, S., 2002. The diagnosis of trypanosome infections: applications of novel technology for reducing disease risk. Afr. J. Biotechnol. 1, 39-45.

Schlater, J., Van den Bossche, P., 2004. Trypanosomosis (Tsetse-Transmitted). OIE Manual of Diagnostic Tests and Vaccines for Terrestrial Animals (Mammals, Birds and Bees) OIE, Paris, pp. 580-588.

Simukoko, H., Marcotty, T., Phiri, I., Geysen, D., Vercruysse, J., Van den Bossche, P., 2007a. The comparative role of cattle, goats and pigs in the epidemiology of livestock trypanosomiasis on the plateau of eastern Zambia. Vet. Parasitol. 147, 231-238.

Simukoko, H., Marcotty, T., Phiri, I., Vercruysse, J., Van den Bossche, P., 2007b. Heterogeneity in the trypanosomosis incidence in Zebu cattle of different ages and sex on the plateau of eastern Zambia. Acta Trop. 103, 98-101.

StataCorp (Ed.), 2006. Stata Statistical Software: Release 9.2. Stata Corporation, Texas.

Swallow (Ed.), 1998. Impacts of African Animal Trypanosomosis on Migration, Livestock and Crop Production. ILRI, Nairobi.

Swallow, B.M., 2000. Impacts of trypanosomiasis on African agriculture. In: PAAT Technical and Scientific Series. FAO, Animal Production and Health Division, International Livestock Research Institute, Nairobi, Kenya, http:// www.fao.org/ag/againfo/programmes/en/paat/documents/papers/Pos2.pdf.

Thrusfield (Ed.), 1995. Veterinary Epidemiology. Blackwell, Oxford.

Trail, J.C.M., D’Ieteren, G.D.M., Murray, M., Ordner, G., Yangari, G., Maille, J.C., Viviani, P., Colardelle, C., Sauveroche, B., 1993. Measurements of trypanotolerance criteria and their effect on reproductive performance of N'Dama cattle. Vet. Parasitol. 45, 241-255.

Van den Bossche, P., Chigoma, D., Shumba, W., 2000. The decline of anti-trypanosomal antibody levels in cattle after treatment with trypanocidal drugs and in the absence of tsetse challenge. Acta Trop. 77, 263-270.

Van den Bossche, P., Rowlands, G.J., 2001. The relationship between the parasitological prevalence of trypanosomal infections in cattle and herd average packed cell volume. Acta Trop. 78, 163-170. 